Revista de Matemática: Teoría y Aplicaciones 2016 23(1) : 1-10

CIMPA - UCR ISSN: 1409-2433 (PRINT), 2215-3373 (ONLINE)

\title{
LOS TEOREMAS DE TYCHONOFF Y DE LOS PRODUCTOS CONEXOS SON EQUIVALENTES
}

\section{TYCHONOFF AND CONNECTED PRODUCTS THEOREMS ARE EQUIVALENT}

\author{
JuAn ANTONIO PÉREZ*
}

Received: 26 Jan 2015; Revised: 28 Aug 2015;

Accepted: 13 Oct 2015

*Cuerpo Académico de Topología y Análisis UAZ 106, Universidad Autónoma de Zacatecas, Zacatecas, México. E-Mail: japerez@uaz.edu.mx 


\title{
Resumen
}

En Topología, el teorema de Tychonoff garantiza la equivalencia entre el hecho de que un producto de espacios topológicos sean compactos y la compacidad de cada uno de los factores, mientras que el teorema de los productos conexos hace lo propio en el caso de la conexidad. En el presente trabajo se demuestra la equivalencia de ambos resultados topológicos.

Palabras clave: compacidad; conexidad; axioma de elección.

\begin{abstract}
In Topology, Tychonoff's theorem asserts that the compactness of a product of topological spaces and compactness of each of its factors are equivalent facts. Analogously, the connected products theorem does the same about connectedness. This note is devoted to prove the equivalence between thes two topological results.
\end{abstract}

Keywords: compactness; connectedness; choice axiom.

Mathematics Subject Classification: 54A02, 54H02, 03E25, $03 E 75$.

\section{Introducción}

En 1930, el matemático ruso Andrey Nikolayevich Tychonnoff, en su célebre artículo [8] de 1930 demostró que el producto arbitrario de espacios topológicos compactos es a su vez compacto, si se le dota de la topología producto. El resultado correspondiente a la propiedad topológica de conexidad parece ser considerado como propio de la cultura matemática media, y sin embargo, no es frecuente encontrar su demostración en los textos de Topología básica. Este importante resultado no tiene ni siquiera la fortuna de contar con un nombre propio, por lo que será identificado en este documento como el teorema de los productos conexos. En su tercera sección de este trabajo se propone una demostración suficientemente elemental de este resultado como para ser incluida en los cursos básicos de Topología.

El matemático norteamericano John Kelley demostró [2] en 1950 la equivalencia del teorema de Tychonoff con el axioma de elección, y en el presente trabajo se propone la equivalencia de este axioma con el teorema de los productos conexos, imitando básicamente el procedimiento usado por Kelley, de donde se obtiene como corolario la equivalencia entre los teoremas de Tychonoff y de los productos conexos. Se caracterizan así estos dos resultados como propios de la matemática cantoriana. 
Dada una colección $\left\{\left(X_{\alpha}, \tau_{\alpha}\right) \mid \alpha \in \mathcal{A}\right\}$ de espacios topológicos, la topología producto $\tau$ sobre el producto cartesiano

$$
X=\prod_{\alpha \in \mathcal{A}} X_{\alpha}
$$

es la topología que tiene como subbase $\mathcal{S}=\left\{p_{\alpha}^{-1}(U) \mid \alpha \in \mathcal{A}, U \in \tau_{\alpha}\right\}$, donde $p_{\alpha}: X \rightarrow X_{\alpha}$ es la proyección obvia. La topología producto de estos espacios es entonces la topología inicial inducida por las proyecciones.

La topología de las cajas sobre $X$ es la topología $\tau_{B}$ que tiene como base la colección de los productos de la forma

$$
U=\prod_{U_{\alpha} \in \tau_{\alpha}} U_{\alpha}
$$

y es claramente más fina que la topología producto, pero ambas coinciden sobre productos con una cantidad finita de factores.

Sea $(X, \tau)$ un espacio topológico, una colección $\mathcal{U} \subseteq 2^{X}$ es una cubierta de $X$ si $\bigcup \mathcal{U}=X$, y es una cubierta abierta si es cubierta y $\mathcal{U} \subseteq \tau$. Si $\mathcal{U}$ es una cubierta para $X$ y $\mathcal{V} \subseteq \mathcal{U}$ también lo es, se dice que $\mathcal{V}$ es una subcubierta de $\mathcal{U}$ para $X$. El espacio topológico $(X, \tau)$ es compacto si toda cubierta abierta para $X$ admite una subcubierta finita.

Una colección $\mathcal{F} \subseteq 2^{X}$ tiene la propiedad de intersección finita (PIF) si toda subcolección finita de $\mathcal{F}$ tiene intersección no vacía. El siguiente resultado es una caracterización de la compacidad por cerrados, y su demostración no requiere sino un uso inmediato de las definiciones.

Proposición 1 Un espacio topológico es compacto si y sólo si toda colección de cerrados de él con la PIF tiene intersección no vacía.

Un par de subespacios abiertos no vacíos $(A, B)$ de un espacio topológico $(X, \tau)$ es una separación si $\{A, B\}$ es una partición de $X$, es decir, si $\{A, B\}$ es una cubierta para $X$ y $A \cap B=\varnothing$. El espacio $(X, \tau)$ es conexo si no admite una separación. Clarametne un espacio no es conexo si y sólo si tiene un subespacio no trivial abierto y cerrado. En el propósito de la autocontención se incluyen el siguiente resultado y un corolario de él que será usado más adelante. Nuevamente, las demostraciones son rutinarias y no se incluyen.

Proposición 2 Si $Y \subseteq X$ es conexo y $Y \subseteq Z \subseteq \bar{Y}$, entonces $Z$ es conexo.

Corolario 3 Si $X$ es un espacio que admite una denso conexo, entonces $X$ es conexo. 


\section{Productos compactos}

En esta sección se demuestra la equivalencia del teorema de Tychonoff con el axioma de elección.

Teorema 4 (Tychonoff, 1930) Un producto es compacto si y sólo si cada uno de los factores es compacto.

Demostración. Sean $\left\{X_{\alpha} \mid \alpha \in \mathcal{A}\right\}$ una colección de espacios topológicos, y $X$ su producto. Si $X$ es compacto, dado que la proyección $p_{\alpha}: X \rightarrow X_{\alpha}$ es contiua y suprayectiva, entonces $X_{\alpha}$ es compacto. Recíprocamente, Sea $\mathcal{F}=\left\{F_{i} \mid i \in\right.$ $\mathcal{I}$ \} una familia de cerrados de $X$ con la PIF, la que puede suponerse maximal, en el sentido de que si $F_{i}, F_{j} \in \mathcal{F}$, entonces $F_{i} \cap F_{j} \in \mathcal{F}$, ya que si $\mathcal{F} \subseteq \mathcal{G}$ y $\cap \mathcal{G} \subseteq \cap \mathcal{F}$. Entonces, para cada $\alpha \in \mathcal{A}$, la colección $\mathcal{F}_{\alpha}=\left\{\overline{p_{\alpha}\left(F_{i}\right)} \mid i \in \mathcal{I}\right\}$ tiene la PIF, de manera que por la compacidad de $X_{\alpha}$, ocurre que $\cap \mathcal{F}_{\alpha} \neq \varnothing$. Elíjase $1 x_{\alpha} \in \cap \mathcal{F}_{\alpha}$, y sea $x=\left(x_{\alpha} \mid \alpha \in \mathcal{A}\right) \in X$, que claramente satisface que $p_{\alpha}(x)=x_{\alpha}$ para cada $\alpha \in \mathcal{A}$. Sea

$$
S=p_{\alpha_{1}}^{-1}\left(U_{\alpha_{1}}\right) \cap \ldots \cap p_{\alpha_{n}}^{-1}\left(U_{\alpha_{n}}\right)
$$

un subbásico de la topología producto para $X$, tal que $x \in S$, entonces $p_{\alpha}(S)$ es un abierto de $X_{\alpha}$ que contiene a $x_{\alpha}$, y en consecuencia $p_{\alpha}(S) \cap p_{\alpha}\left(F_{i}\right) \neq \varnothing$ para todo $\alpha$ y para todo $i$, de manera que $p_{\alpha_{k}}^{-1}\left(U_{\alpha_{k}}\right) \cap F_{i} \neq \varnothing$ para todo $k \in\{1, \ldots, n\}$ y todo $i \in \mathcal{I}$. Sea $y_{\alpha_{k}} \in p_{\alpha_{k}}^{-1}\left(U_{\alpha_{k}}\right) \cap F_{i}$ para $i \in \mathcal{I}$ fijo, y tómese un punto $y \in F_{i}$ tal que $p_{\alpha_{k}}(y)=y_{\alpha_{k}}$, para $k \in\{1, \ldots, n\}$, que claramente satisface que $y \in S \cap F_{i}$, con lo que $S \cap F_{i} \neq \varnothing$. Se sigue de aquí que $S \cap F_{i} \neq \varnothing$, para todo $i \in \mathcal{I}$. Entonces $x \in \overline{F_{i}}=F_{i}$ para todo $i \in \mathcal{I}$, con lo que, entonces $x \in \cap \mathcal{F}$.

En la demostración del resultado anterior juega un papel central el axioma de elección, y es un resultado de Kelley[2], publicado en 1950, que este resultado también implica el axioma de elección.

Teorema 5 (Kelley, 1950) El axioma de elección es equivalente con el Teorema de Tychonoff.

Demostración. Una de las implicaciones ha quedado demostrada en el resultado previo, supongamos entonces válido en teorema de Tychonoff, supóngase que $\mathcal{Y}=\left\{Y_{\alpha} \mid \alpha \in \mathcal{A}\right\}$ es una colección no vacía de conjuntos no vacíos, y sea

$$
y \notin \bigcup_{\alpha \in \mathcal{A}} Y_{\alpha} .
$$

${ }^{1}$ Nótese que aquí se hace uso del axioma de elección. 
Nótese que tal punto en verdad existe, por ejemplo, si

$$
y=\bigcup_{\alpha \in \mathcal{A}} Y_{\alpha} .
$$

Denotemos $X_{\alpha}=Y_{\alpha} \cup\{y\}$, y dótese a este conjunto de la topología $\tau_{\alpha}=$ $\left\{X_{\alpha},\{y\}, \varnothing\right\}$, respecto de la cual es claramente compacto, y por hipótesis, el producto

$$
X=\prod_{\alpha \in \mathcal{A}} X_{\alpha}
$$

es compacto. Claramente $Y_{\alpha}$ es cerrado en $X_{\alpha}$, de manera que por continuidad $p_{\alpha}^{-1}\left(Y_{\alpha}\right)$ es cerrado en $X$ y es no vacío. Para toda subcolección finita $\left\{\alpha_{1}, \ldots, \alpha_{n}\right\} \subseteq \mathcal{A}$ se satisface que

$$
x \in p_{\alpha_{1}}^{-1}\left(Y_{\alpha_{1}}\right) \cap \ldots \cap p_{\alpha_{n}}^{-1}\left(Y_{\alpha_{n}}\right)
$$

donde $x_{\alpha}=y$ para $\alpha \notin\left\{\alpha_{1}, \ldots, \alpha_{n}\right\}$ y se elige $x_{\alpha_{k}} \in Y_{\alpha_{k}}$ para $k \in\{1, \ldots, n\}$ dado que cada $Y_{\alpha}$ es no vacíd 2 . Tenemos entonces que $\left\{p_{\alpha}^{-1}\left(Y_{\alpha}\right) \mid \alpha \in \mathcal{A}\right\}$ es una colección de cerrados de $X$ con la PIF, de manera que por compacidad, tiene intersección no vacía, y dado que

$$
\bigcap_{\alpha \in \mathcal{A}} p_{\alpha}^{-1}\left(Y_{\alpha}\right)=\prod_{\alpha \in \mathcal{A}} Y_{\alpha}
$$

se ha completado la demostración.

\section{Productos conexos}

En esta sección nos proponemos demostrar el resultado análogo al Teorema de Tychonoff para conexidad, resultado al que identificaremos como el teorema de los productos conexos.

Proposición 6 Si cada par de puntos de un espacio X están contenidos en un conjunto conexo, entonces $X$ es conexo.

Demostración. $\mathrm{Si}(A, B)$ es una separación de $X$, tomemos dos puntos $x \in A$, $y \in B$ y un conexo $C \subseteq X$ con $x, y \in C$, entonces $(A \cap C, B \cap C)$ es una separación de $C$.

Este resultado nos permitirá demostrar la conexidad de un producto finito de conexos.

\footnotetext{
${ }^{2}$ Nótese que aquí no es necesario el uso del axioma de elección, dado que se trata de una colección finita; la existencia de cada $x_{\alpha_{k}}$ está garantizada por el axioma de los pares, en la axiomática de Zermelo-Fraenkel.
} 
Lema 7 El producto cartesiano de una familia finita de espacios topológicos es un espacio conexo si y sólo si cada factor es un espacio conexo.

Demostración. Sean $X_{1}, \ldots, X_{n}$ espacios topológicos y $X$ su producto. Si $X$ es conexo, entonces cada factor es conexo por la continuidad de las proyecciones. Demostraremos ahora que si cada factor es conexo, entonces el producto es conexo, iniciando con el producto de dos espacios. Sean $X_{1}, X_{2}$ espacios topológicos conexos, y consideremos dos puntos $x=\left(x_{1}, x_{2}\right), y=$ $\left(y_{1}, y_{2}\right) \in X=X_{1} \times X_{2}$. Basta demostrar que existe un conexo $C \subseteq X$ tal que $x, y \in C$. Si $x_{1}=y_{1}$, entonces basta hacer $C=\left\{\left(x_{1}, z\right) \mid z \in X_{2}\right\}$, que es conexo porque es homeomorfo con $X_{2}$. Si $x_{2}=y_{2}$, entonces basta hacer $C=\left\{\left(z, x_{2}\right) \mid z \in X_{1}\right\}$, que es conexo porque es homeomorfo con $X_{1}$.

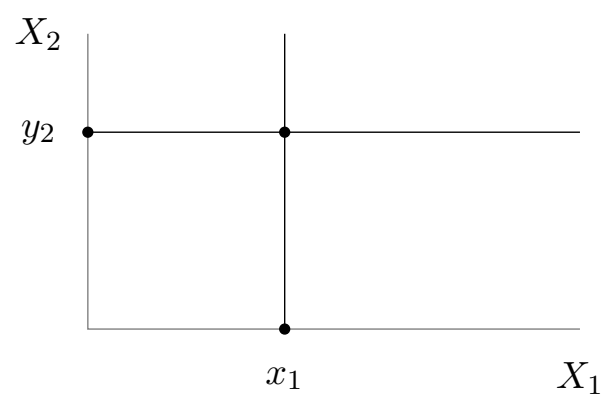

Si los puntos tienen ambas coordenadas distintas, tomemos $z=\left(x_{1}, y_{2}\right)$, entonces, haciendo

$$
C=\left\{\left(x_{1}, z\right) \mid z \in X_{2}\right\} \cup\left\{\left(z, y_{2}\right) \mid z \in X_{1}\right\}
$$

obtenemos el subespacio conexo deseado, puesto que es la unión de dos conexos con un punto común. Por el primer resultado de la sección $X_{1} \times X_{2}$ es conexo. El resultado se cumple para un producto finito arbitrario por inducción sobre el número de factores.

Consideremos ahora un producto arbitrario de espacios topológicos conexos. Sean $\mathcal{X}=\left\{X_{\alpha} \mid \alpha \in \mathcal{A}\right\}$ una colección de espacios topológicos y $X$ su producto. Dados dos puntos $x, y \in X$ definimos $\mathcal{A}_{x, y}=\left\{\alpha \in \mathcal{A} \mid p_{\alpha}(x) \neq p_{\alpha}(y)\right\}$. Decimos que $x, y$ son casi iguales, si $\mathcal{A}_{x, y}$ es finito, lo que denotamos mediante $x \approx y$. Dos puntos entonces son casi iguales si difieren en una cantidad finita de coordenadas, de manera que todos los puntos de un producto finito son casi iguales. Notemos por otra parte que: 
1. $\mathcal{A}_{x, y}=\mathcal{A}_{y, x}$

2. si $x=y$, entonces $x \approx y$

3. $\mathcal{A}_{x, x}=\varnothing$

Para $a \in X$ arbitrario pero fijo, consideremos el subespacio

$$
X_{a}=\{x \in X \mid x \approx a\},
$$

para demostrar que $X$ es conexo, es suficiente demostrar que $X_{a}$ es conexo y denso en $X$.

Demostraremos primero que $X_{a}$ es denso en $X$. Sea $U \subseteq X$ un abierto básico para la topología producto sobre $X$, entonces $U$ tiene la forma

$$
U=p_{\alpha_{1}}^{-1}\left(U_{\alpha_{1}}\right) \cap \ldots \cap p_{\alpha_{n}}^{-1}\left(U_{\alpha_{n}}\right),
$$

donde $U_{\alpha_{k}} \subseteq X_{\alpha_{k}}$ es abierto y no vacío para $k=1, \ldots, n$. Elijamos ahora $x_{\alpha_{k}} \in U_{\alpha_{k}}$ para $k=1, \ldots, n$, y definamos el punto $x \in X$ tal que:

1. $p_{\alpha_{k}}(x)=x_{\alpha_{k}}$ para $k=1, \ldots, n, \mathrm{y}$

2. $p_{\alpha}(x)=a_{\alpha}$ para $\alpha \notin\left\{\alpha_{1}, \ldots, \alpha_{n}\right\}$.

Por construcción es entonces claro que $x \in U$ y $x \approx a$, por lo que $x \in X_{a}$. Queda con ello demostrada la densidad.

Para demostrar la conexidad, sea $x, y \in X_{a}$ dos puntos arbitrarios, y supongamos que $\mathcal{A}_{x, a} \cup \mathcal{A}_{y, a}=\left\{\alpha_{1}, \ldots, \alpha_{n}\right\}$, entonces, por el resultado anterior

$$
Y=X_{\alpha_{1}} \times \ldots \times X_{\alpha_{n}}
$$

es conexo. El espacio $Z$ de los puntos $z \in X_{a}$ tales que:

1. $p_{\alpha_{k}}(z) \in X_{\alpha_{k}}$ para $k=1, \ldots, n, \mathrm{y}$

2. $p_{\alpha}(z)=a_{\alpha}$ para $\alpha \notin\left\{\alpha_{1}, \ldots, \alpha_{n}\right\}$,

es homeomorfo con $Y$ y es tal que $x, y, a \in Z$. Entonces dos puntos cualesquiera de $X_{a}$ están contenidos en un conexo contenido en $X_{a}$, de donde se sigue la conexidad de $X_{a}$.

Teorema 8 (de los productos conexos) El producto cartesiano de una familia de espacios topológicos es un espacio conexo si y sólo si cada factor es un espacio conexo. 
Demostración. Sea $\mathcal{X}=\left\{X_{\alpha} \mid \alpha \in \mathcal{A}\right\}$ una colección de espacios topológicos, y denotemos por $X$ el producto cartesiano de la colección $\mathcal{X}$. Si $X$ es conexo, la continuidad de las proyecciones $p_{\alpha}: X \rightarrow X_{\alpha}$ garantiza la conexidad de cada uno de los factores. Supongamos ahora recíprocamente que $X_{\alpha}$ es un espacio conexo para todo $\alpha \in \mathcal{A}$, para demostrar que $X$ es conexo, basta observar que, con la notación de la discusión previa, $X_{a}$ es conexo y $\overline{X_{a}}=X$ para todo $a \in X$.

\section{La equivalencia}

Teorema 9 El teorema de los productos conexos es equivalente con el axioma de elección.

Demostración. El teorema de los productos conexos es, claramente, consecuencia del axioma de elección. Para demostrar el recíproco, coonsideremos una colección $\mathcal{Y}=\left\{Y_{\alpha} \mid \alpha \in \mathcal{A}\right\}$ una colección no vacía de conjuntos no vacíos, y sea

$$
y \notin \bigcup_{\alpha \in \mathcal{A}} Y_{\alpha}
$$

punto cuya existencia quedó aclarada en la demostración del teorema de Tychonoff. Supondremos que $\mathcal{A}$ es una colección infinita, dado que en el caso finito el resultado es obvio.

Denotemos $X_{\alpha}=Y_{\alpha} \cup\{y\}$, y dótese a este conjunto de la topología $\tau_{\alpha}=$ $\left\{X_{\alpha}, Y_{\alpha},\{y\}, \varnothing\right\}$, respecto de la cual claramente no es conexo, y por hipótesis, entonces el producto

$$
X=\prod_{\alpha \in \mathcal{A}} X_{\alpha}
$$

tampoco es conexo. Denotemos $\mathcal{D}_{x}=\left\{\alpha \in \mathcal{A} \mid p_{\alpha}(x)=y\right\}$, y observemos que si

$$
Y=\prod_{\alpha \in \mathcal{A}} Y_{\alpha}=\bigcap_{\alpha \in \mathcal{A}} p_{\alpha}^{-1}\left(Y_{\alpha}\right)
$$

entonces

$$
Y=\left\{x \in X \mid \mathcal{D}_{x}=\varnothing\right\} .
$$

Supongamos que $Y=\varnothing$, demostraremos que $Y^{c}=X-Y$ es conexo, con lo que quedará establecido que $Y^{c} \neq X$. Sea $\tilde{y} \in X$ tal que $p_{\alpha}(\tilde{y})=y$ para todo $\alpha \in \mathcal{A}$.

Si $(A, B)$ es una separación de $Y^{c}=\left\{x \in X \mid \mathcal{D}_{x} \neq \varnothing\right\}$, se supondrá, sin pérdida de generalidad, que $\tilde{y} \in A$, y sea $V$ un básico de $X$. tal que $\tilde{y} \in V \subseteq A$; 
tal básico existe porque $A \in \mathcal{N}(\tilde{y})$ en $X$. Dada la estructura de la topología producto, $V$ tiene la forma

$$
V=p_{\alpha_{1}}^{-1}(y) \cap \ldots \cap p_{\alpha_{k}}^{-1}(y),
$$

para $\alpha_{1}, \ldots, \alpha_{k} \in \mathcal{A}$, de manera que $V$ contiene al menos un punto $x \in X$ tal que para algún $\alpha \in \mathcal{A}-\left\{\alpha_{1}, \ldots, \alpha_{k}\right\}$ se satisface que $p_{\alpha}(x) \neq y$. Sean $x \in B$ y $U$ un básico tal que $x \in U \subseteq B$, tal básico existe porque $B \in \mathcal{N}(x)$ en $X$. Análogamente observamos que $U$ tiene la forma

$$
U=p_{\alpha_{k+1}}^{-1}(y) \cap \ldots \cap p_{\alpha_{k+i}}^{-1}(y) \cap p_{\beta_{1}}^{-1}\left(Y_{\beta_{1}}\right) \cap \ldots \cap p_{\beta_{m}}^{-1}\left(Y_{\beta_{m}}\right),
$$

para $\alpha_{k+1}, \ldots, \alpha_{k+i}, \beta_{1}, \ldots, \beta_{m} \in \mathcal{A}$. Pero claramente $U \cap V \neq \varnothing$, puesto que, sin recurir al axioma de elección, por tratarse de una colección finita, podemos encontrar un punto $z \in X$ tal que $p_{\beta_{t}}(z) \in Y_{\beta_{t}}$ para $1 \leq t \leq m+j$ y $p_{\alpha}(z)=y$ en cualquier otro caso. Con ello se contradice la hipótesis de que $(A, B)$ sea una separación para $Y^{c}$, y por consiguiente $Y \neq \varnothing$.

Corolario 10 El teorema de los productos conexos es equivalente con el teorema de Tychonoff.

Demostración. Ambos son equivalentes con el axioma de elección.

\section{Agradecimientos}

Es interés del autor manifestar su agradecimiento a los árbitros, sin cuya intervención algunos errores podrían no haber desaparecido. Los que aún estén presentes son exclusivamente responsabilidad propia.

\section{Referencias}

[1] García Máynez, A. (2011) Introducción a la Topología de Conjuntos. Aportaciones Matemáticas No. 36, Sociedad Matemática Mexicana, México D.F.

[2] Kelley, J.L. (1950) "The Tychonoff product theorem implies the axiom of choice”, Fundamenta Mathematica 37: 75-76.

[3] Kelley, J.L. (1975) General Topology. Springer Verlag GTM no. 27, New York. 
[4] Kum, S. (2003) "A correction of Kelley's proof on the equivalence between the Tychonoff product theorem and the axiom of choice", Journal of the Chungcheong Mathematical Society 16(2): 75-78.

[5] Pérez, J.A. (2015) Topología de Conjuntos, un Primer Curso. Publicaciones Electrónicas, Vol. 18, Sociedad Matemática Mexicana, México D.F. http: //sociedadmatematicamexicana.org.mx/SEPA/ ECMS/resumen/P1TE19_1.pdf

[6] Raman-Sundström, M. (2010) "A pedagogical history of compactness", The American Mathematical Monthly 122(7): 619-635.

[7] Salicrup, G. (1993) Introducción a la Topología. Sociedad Matemática Mexicana, Aportaciones Matemáticas, Textos No. 1, México D.F.

[8] Tychonoff, A.N. (1929) “Über die topologische Erweiterung von Räumen”, Mathematische Annalen 102: 544-561. 